\title{
Pseudo-subarachnoid haemorrhage due to chronic hypoxaemia: case report and review of the literature
}

\author{
Maximilian Patzig ${ }^{1 *}$, Christoph Laub ${ }^{2}$, Hendrik Janssen ${ }^{1}$, Lorenz Ertl ${ }^{1}$ and Gunther Fesl ${ }^{1}$
}

\begin{abstract}
Background: The specificity of computed tomography (CT) for subarachnoid haemorrhage $(S A H)$ is very high. However, physicians should be aware of rare false positive findings, also referred to as "pseudo-SAH". We present an unusual case in which such a finding was caused by chronic hypoxaemia.

Case presentation: A 37-year-old male patient presented with headaches. His CT-scan showed multiple confluent subarachnoid hyperattenuations, which mimicked SAH. However, the headache was chronic and had no features typical for SAH. The patient suffered from severe chronic hypoxaemia due to congenital heart failure. On CT-angiography diffuse intracranial vessel proliferation was found and laboratory results revealed a highly raised level of haematocrit, which had both probably developed as compensatory mechanisms. A combination of these findings explained the subarachnoid hyperdensities. Magnetic resonance imaging (MRI) showed no signs of SAH and visualized hypoxaemia in cerebral veins. A diagnosis of pseudo-SAH was made. The patient's symptoms were likely due to a secondary headache attributed to hypoxia and/or hypercapnia. Therapy was symptomatic.
\end{abstract}

Conclusions: Severe chronic hypoxaemia should be recognised as a rare cause of pseudo-SAH. Clinical evaluation and MRI help differentiate SAH from pseudo-SAH.

Keywords: Subarachnoid haemorrhage, Hypoxaemia, Hypoxia, Computed tomography, Magnetic resonance imaging

\section{Background}

In the diagnosis of subarachnoid haemorrhage (SAH), nonenhanced computed tomography (NECT) is known to have a high sensitivity and an even higher specificity, which has recently even been reported to be $100 \%$ [1]. Thus, false positive CT findings, sometimes called pseudo-SAH, are rare. We present an unusual case of pseudo-SAH which had its origin in chronic hypoxaemia.

\section{Case presentation}

A 37-year-old patient had undergone NECT in a radiological practice because of headaches. The scan showed bilateral multiple hyperattenuations in the hemispheral sulci and basal cisterns. A diagnosis of SAH was made and the patient was referred to our emergency department.

\footnotetext{
* Correspondence: Maximilian.Patzig@med.uni-muenchen.de

${ }^{1}$ Department of Neuroradiology, University of Munich - Grosshadern Hospital, Marchioninistr. 15, 81377 Munich, Germany

Full list of author information is available at the end of the article
}

On admission, the patient reported that he had been suffering from progressively worsening headaches for about six months. The pain was of a dull, pressing quality and localized occipitally, radiating to parietal regions bilaterally. The headache was most severe in the morning hours, with a maximum of 7 on a pain scale of $0-10$.

Medical history revealed postnatal major cardiac surgery due to a transposition of the great arteries. Moreover, several pulmonary embolisms were known and the patient was on phenprocoumon as a long-term therapy.

On clinical examination, marked cyanosis, clubbed fingers and hippocratic nails were found, indicating chronic hypoxaemia (Figure 1). The clinical neurological examination was negative. Oxygen saturation measured by pulse oximetry was $70-74 \%$, which the patient said were his "normal" values. Laboratory results notably showed a haematocrit level of $74.8 \%$ and a haemoglobin level of $22.1 \mathrm{mg} / \mathrm{dl}$. International Normalized Ratio (INR) was 8.2 and Partial Thromboplastin Time (PTT) was $114 \mathrm{~s}$. 


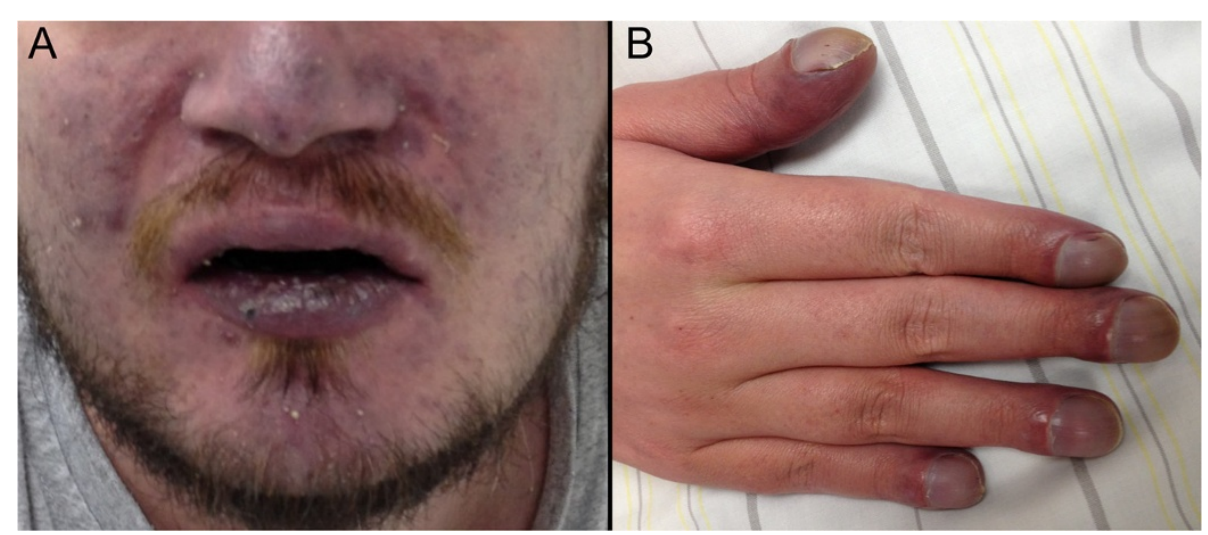

Figure 1 Clinical signs of chronic hypoxaemia. A: Facial cyanosis. B: Clubbed fingers and hippocratic nails.

CT imaging was performed, with the repeated NECT showing the same findings as the scan performed at the external radiology practice (Figure 2A). CT-angiography (CTA) of the intracranial vessels showed no aneurysm or vascular malformation, but dilated veins ubiquitous in the subarachnoid space (Figure 2B). The hyperattenuations on NECT correlated with the course of the vessels found on CTA. Thoracic CT-angiography revealed atrial and ventricular septum defects and a pulmonary trunk fed by both ventricles. The pulmonary arteries were severely dilated, while the aorta, springing regularly from the left ventricle, had a small diameter. The right atrium was enlarged and the right ventricle was hypertrophic. Severe chronic pulmonary thromboembolism was shown.

As the diagnosis of SAH was now strongly doubted, a 3-Tesla MRI scan (Signa HDx scanner, GE, Milwaukee, WI, USA) was performed for further clarification. The protocol included FLAIR, PD and T2*-weighted sequences as well as susceptibility-weighted angiography (SWAN) (Figure 2C and D). SWAN revealed the intracranial veins to be very hypointense and with a large diameter, due to the susceptibility effect caused by the increased fraction of deoxyhaemoglobin in the venous blood of the patient (Figure 2D). The image quality was markedly reduced because of motion artefacts. It was sufficient, however, to rule out the diffuse CThyperattenuations representing subarachnoid blood. There were no findings indicative of acute $\mathrm{SAH}$ or superficial haemosiderosis.

In view of these findings, the diagnosis of diffuse $\mathrm{SAH}$ was rejected. We therefore refrained from a lumbar puncture (particularly in view of the impaired coagulation) or digital subtraction angiography (DSA). The patient obviously had chronic hypoxaemia due to the complex cardiopulmonary situation with major intermixture of the blood of the systemic and pulmonary circulations. Compensatory polycythaemia had developed. Generalized proliferation and dilatation of intracranial veins likely were another compensatory mechanism to maintain sufficient cerebral oxygen supply. The increased haematocrit in combination with the prominent vessels can explain the subarachnoid hyperdensities, which mimicked severe SAH. The headache had no features characteristic of SAH. It was classified as chronic tension headache or headache in relation to hypoxia and/or hypercapnia. A symptomatic therapy was recommended.

\section{Conclusions}

Hyperattenuations of the sulci and/or basal cisterns on NECT in most cases represent SAH. In some patients, however, the underlying pathology is different. These cases are also referred to as "pseudo-SAH". This phenomenon has been recognized in the literature in several case reports and rather small case series. In rare cases, leptomeningeal disesases such as purulent meningitis [2,3], meningeal leukaemia [4] and gliomatosis [5] have been reported to mimic SAH. Contrast agent neurotoxicity is another rare cause of CT hyperdensities which can be mistaken for $\mathrm{SAH}$. Obviously, however, this can only be a differential diagnosis if contrast has been applied, usually via DSA [6-8]. Moreover, pseudo-SAH has been encountered in patients with intracranial hypotension [9-11], cerebellar infarctions $[12,13]$ and bilateral subdural haematoma $[14,15]$. Most reports of pseudo-SAH, however, exist on patients with severe generalized cerebral edema. These include rare cases of toxic encephalopathy after valproic acid [16] or bee sting poisoning [17], but mostly patients with severe hypoxic or anoxic encephalopathy [18-25]. Avrahami et al. [25] performed lumbar punctures in 26 comatose patients with generalized cerebral edema and CT diagnosis of SAH, and found no bloody or xanthochromic cerebrospinal fluid in any of them. Yuzawa et al. [18] report pseudo-SAH in eight of 45 consecutive patients who underwent CT scans after resuscitation from cardiopulmonary arrest. PseudoSAH was associated with severe cerebral edema in all of these patients. Explanations for the paradoxical imaging 


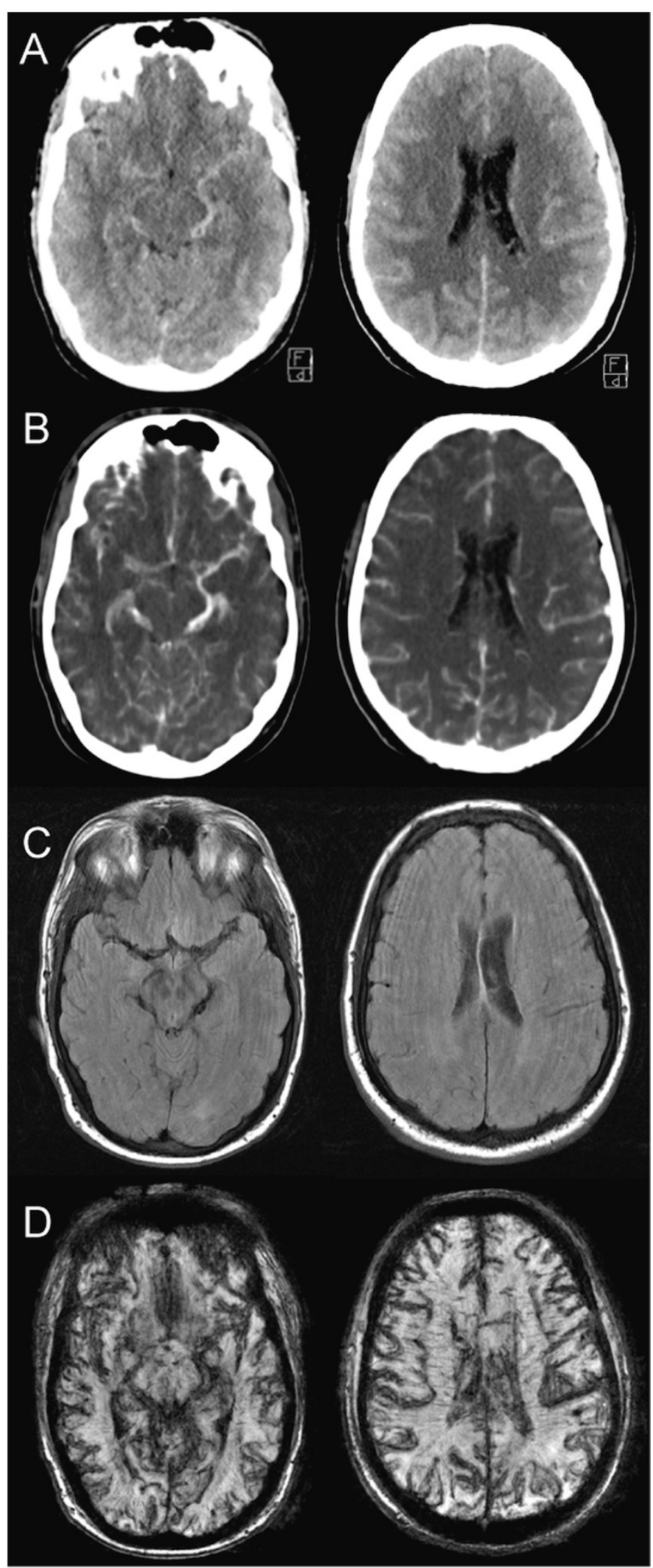

Figure $\mathbf{2}$ (See legend on next page.) 
(See figure on previous page.)

Figure 2 Imaging. Exemplary images of matching slice plains. All images represent $5 \mathrm{~mm}$ slices, with images in A, B and D being

reconstructions of thinner source images. MR images are of reduced quality due to motion artefacts. A: Non-enhanced CT: Diffuse subarachnoid hyperattenuations mimicking SAH. B: CT-angiography: Sulcal and basal veins dilated and increased in number. C: MRI, FLAIR sequence: No findings of SAH. D: MRI, SWAN sequence: Markedly hypointense depiction of cerebral and subarachnoidal veins due to hypoxaemia.

findings include a narrowing of the sulci and cisterns and a shift of cortex into the subarachnoid spaces, along with pathologically reduced density of adjacent brain parenchyma and vessel congestion.

In our case of pseudo-SAH, we believe the origin of the hyperattenuations in the subarachnoid space to be different from the reports mentioned above, which mostly describe acute encephalopathies. Our patient suffered from chronic hypoxaemia, and there were no signs of cerebral edema on the CT and MRI scans. So there was no thinning of the sulci and no loss of density of the cortex which could explain the sulcal hyperdensities. We believe that one reason for the hyperattenuations in the CT scan of our patient was his extremely elevated haematocrit level. The density of intravascular blood correlates linearly positively with the haematocrit level [26-30]. Increased vessel density due to polycythaemia is known to be a pitfall in the diagnosis of hyperdense middle cerebral artery signs $[31,32]$ or venous sinus thrombosis [33,34]. Brief reports also exist on diffuse vessel hyperdensities caused by polycythaemia $[26,35]$. The other major factor in our case were proliferated and dilated sulcal and basal veins, which likely had developed to compensate for the chronic hypoxaemia. So we hypothesize that numerous and prominent vessels which appeared hyperdense because of a highly raised haematocrit level had caused the SAH-like picture.

The use of Hounsfield unit (HU) measurements to discriminate between pseudo-SAH and SAH in patients with post-resuscitation encephalopathy has been discussed, with lower $\mathrm{HU}$ values presumed to indicate pseudo-SAH [18]. Our patient had relatively high HU values of $52-62$ (measured in the sylvian fissure). However, given the different aetiology of pseudo-SAH in our case and the haematocrit level of $75 \%$, we did not interpret these measurements as favouring a diagnosis of SAH.

The case illustrates a very rare differential diagnosis of SAH on CT scans. It should be considered particularly if the clinical symptoms are not typical for SAH and the patient has severe polycythaemia. When such a case is suspected, we suggest imaging like CT-angiography and particularly MRI to confirm the diagnosis. In low-risk patients, a lumbar puncture to definitely exclude $\mathrm{SAH}$ should be discussed. In our severely coagulopathic patient, however, we wanted to avoid invasive diagnostics. The MRI scan had shown that the diffuse hyperdensities found on NECT did not represent blood. This imaging finding along with the clinical picture, which was not indicative of $\mathrm{SAH}$, gave us enough diagnostic confidence to refrain from invasive methods.

The case also underlines the ability of the SWAN MRI sequence to visualize a reduced oxygen content in cerebral veins. The veins appeared markedly hypointense in a patient with obvious severe chronic hypoxaemia. Susceptibility-weighted imaging (SWI) is known to be sensitive to different oxygenation statuses [36,37], and we found that SWAN, a T2\%-weighted multiple-echo gradient-echo sequence which differs from SWI by using only magnitude information, also has that quality. Further studies are needed to explore clinical applications of this potential.

The headache of our patient fulfilled the criteria of chronic tension headache. However, considering the underlying disorders of the patient and the associations of headaches with polycythaemia, chronic lung diseases and hypoxia as experienced at great altitude [38-42], a secondary headache attributed to hypoxia/hypercapnia with the phenotype of tension headache was the more likely diagnosis. Either way, therapy could only be symptomatic.

\section{Consent}

Written informed consent was obtained from the patient for publication of this case report and any accompanying images. A copy of the written consent is available for review by the Editor of this journal.

\section{Competing interests}

The authors declare that they have no competing interests.

\section{Authors' contributions}

MP was involved in the imaging diagnostics of the patient, performed literature research and drafted the manuscript. CL was involved in the clinical care of the patient, provided clinical information and revised the manuscript. $\mathrm{HJ}$ and LE were involved in the imaging diagnostics of the patient and revised the manuscript. GF was involved in the imaging diagnostics of the patient, conceived the report structure and revised the manuscript. All authors read and approved the final manuscript.

\section{Acknowledgements}

We thank Ms. Katie Ogston for language editing.

\section{Author details}

'Department of Neuroradiology, University of Munich - Grosshadern Hospital, Marchioninistr. 15, 81377 Munich, Germany. 'Department of Neurology, University of Munich - Grosshadern Hospital, Marchioninistr. 15, 81377 Munich, Germany.

Received: 3 August 2014 Accepted: 4 November 2014

Published online: 18 November 2014 


\section{References}

1. Perry JJ, Stiell IG, Sivilotti ML, Bullard MJ, Emond M, Symington C, Sutherland J, Worster A, Hohl C, Lee JS, Eisenhauer MA, Mortensen M, Mackey D, Pauls M, Lesiuk H, Wells GA: Sensitivity of computed tomography performed within six hours of onset of headache for diagnosis of subarachnoid haemorrhage: prospective cohort study. BMJ 2011, 343:d4277

2. Mendelsohn DB, Moss ML, Chason DP, Muphree S, Casey S: Acute purulent leptomeningitis mimicking subarachnoid hemorrhage on $\mathrm{CT}$. J Comput Assist Tomogr 1994, 18(1):126-128.

3. Nakae $Y$, Kudo Y, Yamamoto R, Johkura K: Pseudo-subarachnoid hemorrhage in cryptococcal meningitis: MRI findings and pathological study. Neurol Sci 2013, 34(12):2227-2229.

4. Hsieh SW, Khor GT, Chen CN, Huang P: Pseudo subarachnoid hemorrhage in meningeal leukemia. J Emerg Med 2012, 42(5):e109-e111.

5. Belsare G, Lee AG, Maley J, Kirby P, St Louis EK, Follett K: Pseudosubarachnoid hemorrhage and cortical visual impairment as the presenting sign of gliomatosis cerebri. Semin Ophthalmol 2004 19(3-4):78-80.

6. Ko DY: Contrast agent neurotoxicity presenting as subarachnoid hemorrhage. Neurology 2000, 54(4):1014-1015.

7. Velden J, Milz P, Winkler F, Seelos K, Hamann GF: Nonionic contrast neurotoxicity after coronary angiography mimicking subarachnoid hemorrhage. Eur Neurol 2003, 49(4):249-251.

8. Potsi S, Chourmouzi D, Moumtzouoglou A, Nikiforaki A, Gkouvas K, Drevelegas A: Transient contrast encephalopathy after carotid angiography mimicking diffuse subarachnoid haemorrhage. Neuro/ $\mathrm{SCl}$ 2012, 33(2):445-448

9. Balgera R, Rigamonti A, Agostoni E: Images from headache: spontaneous intracranial hypotension presenting as "pseudo" subarachnoid hemorrhage. Headache 2008, 48(4):614-615

10. Schievink WI, Maya MM, Tourje J, Moser FG: Pseudo-subarachnoid hemorrhage: a CT-finding in spontaneous intracranial hypotension. Neurology 2005, 65(1):135-137.

11. Ferrante E, Regna-Gladin C, Arpino I, Rubino F, Porrinis L, Ferrante MM, Citterio A: Pseudo-subarachnoid hemorrhage: a potential imaging pitfall associated with spontaneous intracranial hypotension. Clin Neurol Neurosurg 2013, 115(11):2324-2328

12. Barton BR, Prabhakaran S, Lopes DK, Lee VH: Pseudo-subarachnoid hemorrhage in cerebellar infarction. Neurocrit Care 2007, 7(2):172-174.

13. Misra V, Hoque R, Gonzalez-Toledo E, Kelley RE, Minagar A: Pseudosubarachnoid hemorrhage in a patient with acute cerebellar infarction. Neurol Res 2008, 30(8):813-815

14. Huang D, Abe T, Ochiai S, Kojima K, Tanaka N, Zhang Y, Nishimura H, Hayabuchi N, Norbash AM: False positive appearance of subarachnoid hemorrhage on CT with bilateral subdural hematomas. Radiat Med 1999 17(6):439-442

15. Rabinstein AA, Pittock SJ, Miller GM, Schindler JJ, Wijdicks EF: Pseudosubarachnoid haemorrhage in subdural haematoma. J Neurol Neurosurg Psychiatry 2003, 74(8):1131-1132.

16. Min YG, Tse ML: Image in toxicology: Pseudo-subarachnoid hemorrhage in a case of severe valproic acid poisoning. Clin Toxicol (Phila) 2011, 49(7):699-700.

17. Yasar Tekelioglu U, Demirhan A, Akkaya A, Gurel K, Ocak T, Duran A, Kocoglu H: Pseudo-subarachnoid haemorrhage and death after bee sting. Med Glas (Zenica) 2013, 10(1):182-185.

18. Yuzawa H, Higano S, Mugikura S, Umetsu A, Murata T, Nakagawa A, Koyama A, Takahashi S: Pseudo-subarachnoid hemorrhage found in patients with postresuscitation encephalopathy: characteristics of CT findings and clinical importance. AJNR Am J Neuroradiol 2008, 29(8):1544-1549.

19. Agha A, Al-Hakami M: A case report of pseudo-subarachnoid hemorrhage. Maedica (Buchar) 2011, 6(3):210-212

20. Given CA 2nd, Burdette JH, Elster AD, Williams DW 3rd: Pseudo-subarachnoid hemorrhage: a potential imaging pitfall associated with diffuse cerebral edema. AJNR Am J Neuroradiol 2003, 24(2):254-256.

21. Spiegel SM, Fox AJ, Vinuela F, Pelz DM: Increased density of tentorium and falx: a false positive CT sign of subarachnoid hemorrhage. Can AssoC Radiol J 1986, 37(4):243-247.

22. al-Yamany M, Deck J, Bernstein M: Pseudo-subarachnoid hemorrhage: a rare neuroimaging pitfall. Can J Neuro/ Sci 1999, 26(1):57-59.
23. Chute DJ, Smialek JE: Pseudo-subarachnoid hemorrhage of the head diagnosed by computerized axial tomography: a postmortem study of ten medical examiner cases. J Forensic Sci 2002, 47(2):360-365.

24. Ahn JH, Choi SC, Jung YS, Min YG: Clinical characteristics of patients with pseudo-subarachnoid haemorrhage who were successfully resuscitated from out-of-hospital cardiopulmonary arrest. Hong Kong J Emerg Med 2012, 19(2):85-91.

25. Avrahami $E$, Katz $R$, Rabin $A$, Friedman $V: C T$ diagnosis of non-traumatic subarachnoid haemorrhage in patients with brain edema. Eur J Radiol 1997, 28:222-225

26. Ben Salem D, Osseby GV, Rezaizadeh-Bourdariat K, Pastural G, Martin D, Brunotte F, Moreau T, Giroud M, Binnert D: Spontaneous hyperdense intracranial vessels seen on CT scan in polycythemia cases. J Radio/ 2003, 84(5):605-608.

27. Black DF, Rad AE, Gray LA, Campeau NG, Kallmes DF: Cerebral venous sinus density on noncontrast CT correlates with hematocrit. AJNR Am J Neuroradiol 2011, 32(7):1354-1357.

28. Collins AJ, Gillespie S, Kelly BE: Can computed tomography identify patients with anaemia? Ulster Med J 2001, 70(2):116-118.

29. Lee SY, Cha SH, Lee SH, Shin DI: Evaluation of the effect of hemoglobin or hematocrit level on dural sinus density using unenhanced computed tomography. Yonsei Med J 2013, 54(1):28-33.

30. New PF, Aronow S: Attenuation measurements of whole blood and blood fractions in computed tomography. Radiology 1976, 121(3 Pt. 1):635-640

31. Koo CK, Teasdale E, Muir KW: What constitutes a true hyperdense middle cerebral artery sign? Cerebrovasc Dis 2000, 10(6):419-423.

32. Rauch RA, Bazan C 3rd, Larsson EM, Jinkins JR: Hyperdense middle cerebral arteries identified on CT as a false sign of vascular occlusion. AJNR Am J Neuroradiol 1993, 14(3):669-673.

33. Besachio DA, Quigley EP 3rd, Shah LM, Salzman KL: Noncontrast computed tomographic Hounsfield unit evaluation of cerebral venous thrombosis: a quantitative evaluation. Neuroradiology 2013, 55(8):941-945.

34. Buyck PJ, De Keyzer F, Vanneste D, Wilms G, Thijs V, Demaerel P: CT density measurement and $\mathrm{H}: \mathrm{H}$ ratio are useful in diagnosing acute cerebral venous sinus thrombosis. AJNR Am J Neuroradiol 2013, 34(8):1568-1572.

35. Javedan SP, Marciano F: Pseudo-enhancement from polycythemia. Neurology 2004, 62(1):150.

36. Ogawa S, Lee TM, Kay AR, Tank DW: Brain magnetic resonance imaging with contrast dependent on blood oxygenation. Proc Natl Acad Sci U S A 1990, 87(24):9868-9872.

37. Rauscher A, Sedlacik J, Barth M, Haacke EM, Reichenbach JR: Nonnvasive assessment of vascular architecture and function during modulated blood oxygenation using susceptibility weighted magnetic resonance imaging. Magn Reson Med 2005, 54(1):87-95.

38. Aamodt AH, Borch-lohnsen B, Hagen K, Stovner $\amalg$, Asberg A, Zwart JA: Headache prevalence related to haemoglobin and ferritin. HUNT Study Cephalalgia 2004, 24(9):758-762.

39. Aamodt AH, Stovner $L$, Langhammer A, Hagen K, Zwart JA: Is headache related to asthma, hay fever, and chronic bronchitis? The Head-HUNT Study. Headache 2007, 47(2):204-212.

40. Arregui A, Leon-Velarde F, Cabrera J, Paredes S, Vizcarra D, Umeres H: Migraine, polycythemia and chronic mountain sickness. Cephalalgia 1994 14(5):339-341.

41. Lopez Jl, Holdridge A, Mendizabal JE: Altitude headache. Curr Pain Headache Rep 2013, 17(12):383.

42. Ozge A, Ozge C, Kaleagasi $\mathrm{H}$, Yalin OO, Unal O, Ozgur ES: Headache in patients with chronic obstructive pulmonary disease: effects of chronic hypoxaemia. J Headache Pain 2006, 7(1):37-43.

\section{doi:10.1186/s12883-014-0219-7}

Cite this article as: Patzig et al:: Pseudo-subarachnoid haemorrhage due to chronic hypoxaemia: case report and review of the literature. BMC Neurology 2014 14:219. 